\title{
Impacto do Tráfego Não Uniforme no Desempenho dos Algoritmos de Roteamento Fixo em Redes Ópticas Transparentes
}

\author{
Gilvan M. Durães, André C. B. Soares e William F. Giozza
}

\begin{abstract}
Resumo- A maioria dos trabalhos sobre redes ópticas WDM transparentes comutadas por circuitos considera como técnica de roteamento fixo, um algoritmo de menor caminho, como o algoritmo de Dijkstra (DJK). Contudo, recentemente, foram propostos dois novos algoritmos, o Restricted Routing Technique (RRT) e o Melhor entre as Menores Rotas (MMR), que buscam um melhor balanceamento da carga e apresentam desempenho superior ao de Dijkstra quando submetidos a uma carga de tráfego distribuída uniformemente pelos nós da rede. Este trabalho avalia o desempenho destes mesmos algoritmos agora sob condições de uma carga de tráfego distribuída não uniformemente pelos nós da rede. Para cada topologia estudada foram observadas as características peculiares do impacto do tráfego não uniforme segundo o algoritmo de roteamento utilizado. De maneira geral, observou-se que o algoritmo MMR apresenta um desempenho superior em termos de probabilidade de bloqueio e justiça para todas as topologias de rede estudadas.
\end{abstract}

Palavras-Chave - Redes Ópticas Transparentes, Roteamento Fixo, Avaliação de Desempenho, Tráfego Não Uniforme.

Abstract-Most works about all-optical circuit-switched WDM networks considers a shortest path algorithm for fixed routing, like the Dijkstra' algorithm. However, recently, two new algorithms, searching for better load balancing and presenting superior performance than Dijkstra's when submitted to an uniform traffic load, were proposed, the Restricted Routing Technique (RRT) and the Melhor entre as Menores Rotas (MMR). This work evaluates the performance of these algorithms under a non-uniform traffic load. For each topology studied were observed specific characteristics of the impact of the non-uniform traffic load for each routing algorithm. Overall, performance evaluation results showed that the algorithm MMR achieves for all topologies studied superior performance in terms of blocking probability and fairness.

Keywords- All-Optical Networks, Fixed Routing, Performance Evaluation, Non-Uniform Traffic.

\section{INTRODUÇÃO}

Cada vez mais os diversos serviços existentes ou emergentes na Internet, como televisão via Internet, vídeo sob demanda, aplicações peer-to-peer e redes privadas virtuais, impulsionam uma crescente demanda de banda

Gilvan M. Durães, gilvan.duraes@unifacs.br, Universidade Salvador (UNIFACS), Núcleo de Pesquisa em Redes e Computação (NUPERC), Salvador, BA, Brasil.

William F. Giozza, giozza@ene.unb.br, Universidade de Brasília (UnB), Departamento de Engenharia Elétrica (EnE), Brasília, DF, Brasil.

André C. B. Soares, andre.soares@ufpi.br, Universidade Federal do Piauí (UFPI), Departamento de Informática e Estatística, Teresina, PI, Brasil.

Gilvan M. Durães é aluno de mestrado do PPGCOMP/UNIFACS, bolsista CAPES passante nos backbones dos sistemas de comunicações [1]. Neste cenário, a tecnologia de redes ópticas é apontada como um dos principais veículos para suportar tal evolução.

A tecnologia Wavelength Division Multiplexing (WDM) permite a multiplexação de uma fibra óptica em diferentes canais ópticos representados por diferentes comprimentos de onda. Assim, a tecnologia WDM possibilita o estabelecimento de mais de um circuito óptico de forma paralela em uma mesma fibra óptica. Essa característica representa uma capacidade em atender maiores demandas de tráfego aproveitando-se de uma infraestrutura e investimentos já realizados [2].

As redes ópticas podem ser classificadas como opacas ou transparentes. Nas redes ópticas opacas o roteamento de comprimento de onda é realizado no domínio eletrônico fazendo uso de conversores Opto-Eletro-Óptico (OEO). Já nas redes ópticas transparentes o roteamento é realizado no nível óptico. Isso permite eliminar os conversores OEO e os impactos negativos do seu uso, e.g. aumento no custo dos equipamentos e inserção de atrasos de processamento [2]. Por isso, o uso da tecnologia de redes ópticas transparentes nas redes de transporte pode ser vista como uma tendência.

Para o estabelecimento de um circuito óptico entre um nó origem e um nó destino, é necessário determinar uma rota e alocar um comprimento de onda em cada enlace da rota escolhida. O problema de roteamento e alocação de comprimento de onda é conhecido como Routing and Wavelength Assignment - RWA [3]. Tipicamente o comprimento de onda alocado deve ter continuidade, isto é, ser o mesmo em todos os enlaces da rota. Esta propriedade é conhecida como continuidade obrigatória de comprimento de onda [3].

Para evitar a propriedade de continuidade obrigatória de comprimento de onda, os nós intermediários em uma determinada rota devem ter a capacidade de converter um comprimento de onda de entrada em um comprimento de onda diferente de saída. Isso é possível com o uso de conversores de comprimento de onda nestes nós intermediários. Todavia, a tecnologia destes dispositivos ainda é pouco amadurecida e o seu custo é elevado. Por isso, este trabalho considera apenas as redes WDM típicas, que respeitam a propriedade da continuidade obrigatória de comprimento de onda, ou seja, redes ópticas sem capacidade de conversão de comprimento de onda.

O problema RWA pode considerar tráfego estático ou dinâmico [3]. No problema RWA estático é definida previamente uma matriz de tráfego que representa o conjunto de circuitos ópticos que devem ficar ativos por um período indeterminado. Neste caso, os circuitos ópticos são 
estabelecidos offline e o objetivo na solução do problema RWA estático é minimizar número de comprimentos de onda necessário para atendê-los. O problema RWA dinâmico, por sua vez, deve ser resolvido com a rede óptica em operação. Neste contexto de maior dinamismo, requisições de circuitos ópticos chegam sob demanda. Por isso a escolha de uma rota e a alocação de um comprimento de onda são realizados on-line, isto é, com a rede óptica em operação. Caso não haja recursos suficientes para atender a uma determinada requisição de circuito óptico, esta requisição será bloqueada. Por esta razão, o objetivo do problema RWA dinâmico é atender às requisições de circuito óptico visando minimizar a probabilidade de bloqueio de futuras demandas de circuitos ópticos.

Este trabalho considera a operação de redes ópticas transparentes num cenário com RWA dinâmico por ser o mais flexível e adequado às características das modernas redes ópticas de transporte. No RWA dinâmico os algoritmos de roteamento podem ser separados em três classes: roteamento fixo, roteamento alternativo e roteamento exaustivo [2].

No roteamento fixo, cada par de nós (origem, destino) da rede óptica dispõe de apenas uma rota que é computada previamente. Assim, antes mesmo de surgir uma requisição de circuito óptico, o Plano de Controle responsável pelo roteamento já sabe qual rota deve ser utilizada. Isto significa que após o surgimento da requisição, o desafio passa a ser a alocação de um comprimento de onda. A maioria dos trabalhos na literatura especializada sobre o problema RWA em redes ópticas transparentes (e.g. $[3,5,6])$ considera o uso de algoritmos de menor caminho (menor número de saltos, isto é, o custo de cada enlace é igual a 1) para definir uma rota fixa para cada par de nós (origem, destino). Dentre os algoritmos de menor caminho, o algoritmo de Dijkstra [4] é um dos mais citados. Entretanto, outros algoritmos têm sido propostos com o objetivo de melhorar o desempenho de uma rede óptica transparente, em termos de probabilidade de bloqueio de requisições de circuitos ópticos com relação ao obtido usando Dijkstra. Dentre estes, destacam-se por exemplo o algoritmo Restricted Routing Technique (RRT) [7] e o algoritmo Melhor entre as Menores Rotas (MMR) [8].

No roteamento alternativo, um conjunto com mais de uma rota é definido previamente para cada par de nós (origem, destino). Isto representa mais de uma alternativa, em termos de rota, na tentativa de estabelecer um circuito óptico $[2,5,6]$.

Os algoritmos da classe de roteamento exaustivo têm como vantagem a capacidade de utilizar qualquer rota possível da topologia na tentativa de estabelecer um circuito óptico [2]. Com isso, uma requisição de circuito óptico apenas será bloqueada se nenhuma rota, entre sua origem e destino, dispuser de pelo menos um comprimento de onda contínuo livre.

Em termos gerais, as classes de algoritmos de roteamento apresentam a seguinte ordem crescente de desempenho em termos de probabilidade de bloqueio: fixo, fixo alternativo e exaustivo $[2,3]$. Entretanto, esse aumento do desempenho é acompanhado também pelo incremento da complexidade de suas soluções. Algoritmos de roteamento que dependem de informações globais sobre o estado da rede resultam em uma maior complexidade para os protocolos do Plano de Controle. Tal complexidade pode ser traduzida em um maior atraso no estabelecimento de um circuito óptico. Certamente, o cálculo prévio das rotas, com o uso de um algoritmo representante da classe de roteamento fixo, potencializa a redução do tempo requerido para o estabelecimento dinâmico do circuito óptico. Além disso, o roteamento fixo implica numa menor complexidade para os protocolos do Plano de Controle, uma vez que a escolha da rota para cada par de nós origem e destino é feita off-line em uma fase de planejamento da rede óptica.

Este trabalho tem como objetivo analisar o impacto de cargas de tráfego não uniforme no desempenho dos algoritmos de roteamento fixo em redes ópticas transparentes sob diferentes topologias.

O restante do artigo está organizado da seguinte forma. Na próxima Seção são apresentados os algoritmos da classe de roteamento fixo. Na Seção III é realizado o estudo de avaliação de desempenho que analisa o impacto do tráfego não uniforme nestes algoritmos. Por último, as conclusões são feitas na Seção IV.

\section{ALGORITMOS DE ROTEAMENTO FIXO EM REDES ÓPTICAS TRANSPARENTES}

Esta Seção apresenta algumas considerações referentes ao algoritmo clássico de menor caminho e uma breve descrição dos algoritmos RRT e MMR.

A partir daqui neste artigo, é utilizada a notação $\langle a, b\rangle$ para indicar um enlace bidirecional que conecta diretamente os nós $a$ e $b$. Ao tempo em que o termo "menor caminho" é utilizado para denotar um menor caminho em termos de número de saltos em uma rota.

\section{A. Algoritmos Clássicos de Menor Caminho}

Os algoritmos clássicos de menor caminho têm como objetivo encontrar um menor caminho qualquer entre dois nós (origem, destino), dos quais o mais referenciado é o algoritmo de Dijkstra [4]. No entanto, podem existir mais de um menor caminho entre dois nós de uma rede. Por exemplo, na topologia A6NET [8], ilustrada na Figura 1, existem 3 menores caminhos para o par $(1,4)$, cada um com três saltos. São eles: 1-2-3-4, 1-2-5-4 e 1-2-6-5-4. Desta forma, existem $\mathrm{M}$ soluções diferentes de rotas de menores caminhos em uma determinada topologia e cada solução desta pode levar a diferentes níveis de desempenho, em termos de probabilidade de bloqueio geral da rede [8]. Contudo, os algoritmos clássicos de menor caminho encontram qualquer uma dessas soluções de menor caminho, sem nenhum critério a mais, além do número de saltos das rotas.

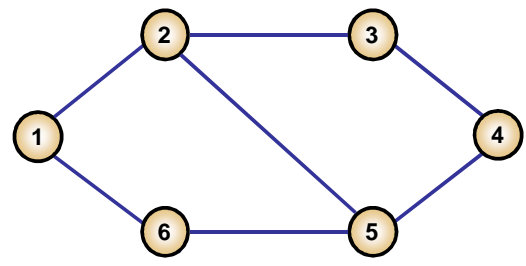

Fig. 1. Topologia A6NET. 


\section{B. Restricted Routing Technique - RRT}

Este algoritmo busca um melhor balanceamento da carga de tráfego procurando aliviar alguns enlaces da rede, em termos de número de rotas que os atravessam. Uma lista de pares de nós (origem, destino) é percorrida, escolhendo rotas para cada par. Durante este percurso, enlaces críticos (enlaces que possuem maior quantidade de rotas já definidas) são retirados temporariamente visando escolher rotas disjuntas destes enlaces. É perceptível que, por causa destas remoções de enlaces, o RRT pode escolher rotas que não sejam de menor caminho. Seus autores o avaliaram apenas em cenários de rede com a capacidade de conversão total de comprimento de onda. Maiores detalhes do RRT podem ser encontrados em [7].

\section{Melhor entre as Menores Rotas - MMR}

O algoritmo MMR faz uso do algoritmo de Dijkstra de forma iterativa, onde a cada iteração, o objetivo é diminuir a probabilidade de bloqueio. Para alcançar este objetivo, a cada iteração os custos dos enlaces são levemente alterados, visando encontrar novas rotas de menor caminho que equilibrem a utilização de todos os enlaces da rede. Os autores do MMR o avaliaram comparativamente com os algoritmos de Dijkstra e RRT, sob as condições de tráfego uniforme [8].

\section{ANÁLISE DO IMPACTO DO TRÁFEGO NÃO UNIFORME NOS ALGORITMOS}

A análise do impacto do tráfego não uniforme nos algoritmos de roteamento fixo foi realizada com o auxílio da ferramenta de simulação TONetS [9]. A seguir é explicado como foi modelado o tráfego não uniforme. Em seguida, são apresentadas as características dos cenários de simulação e, por fim, os resultados obtidos são analisados e discutidos.

\section{A. Modelagem do Tráfego Não Uniforme}

A carga de tráfego considerada neste trabalho segue o modelo Population-Distance (PD) [10]. Aplicando este modelo, o tráfego gerado na rede é dividido de forma ponderada para cada par de nós (origem, destino) de acordo com a Equação 1.

$$
\text { PesoTráfego } o_{\text {Origem,Destino }}=\frac{\text { Pop }_{\text {Origem }} \text { Pop }_{\text {Destino }}}{\text { Dist }_{\text {Origem,Destino }}}
$$

onde, Pop indica a população em milhões de habitantes e Dist indica a distância em kilômetros entre a origem e o destino de um circuito óptico.

\section{B. Características dos Cenários de Simulação}

A demanda de tráfego é composta por requisições de circuitos ópticos representados por pares de nós (origem, destino). É assumido que cada requisição atendida de circuito óptico utiliza dois circuitos ópticos (um no sentido de ida e outro no sentido de volta). A geração de requisições é um processo poissoniano de taxa média $\lambda$ e o tempo médio de retenção dos circuitos é distribuído exponencialmente com média $1 / \mu$; a intensidade de tráfego na rede em Erlangs é dada por $\rho=\lambda / \mu$. Todos os enlaces da rede são bidirecionais e possuem 40 comprimentos de onda em cada sentido. O algoritmo First-Fit [3] é utilizado na alocação dos comprimentos de onda. Para cada simulação são realizadas cinco replicações com diferentes sementes de geração de variável aleatória. São geradas cinco milhões de requisições para cada replicação. Os resultados gráficos apresentam os intervalos de confiança calculados com um nível de confiança de $95 \%$.

Nessa avaliação são utilizadas as topologias das redes RNP [11], EON [12] e Abilene [13]. É avaliado o desempenho dos algoritmos Dijkstra [4] (DJK), RRT [7] e MMR [8].

\section{Discussão dos Resultados}

Inicialmente são apresentados os resultados sob o tráfego uniforme apenas para ratificar a avaliação de desempenho feita em [8], na qual apresentou resultados apenas para duas topologias. As Figuras 2 a 4 mostram os gráficos de probabilidade de bloqueio para as topologias RNP, EON e Abilene, respectivamente.

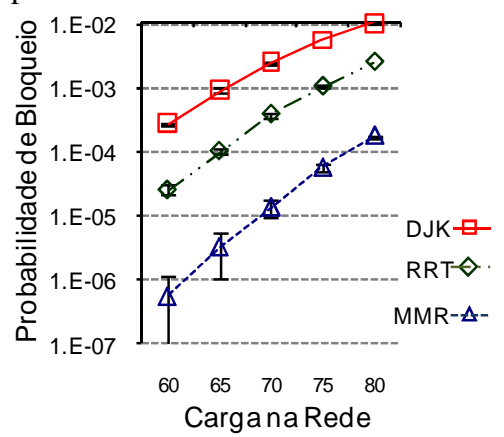

Fig. 2. Probabilidade de Bloqueio, RNP - Tráfego Uniforme.

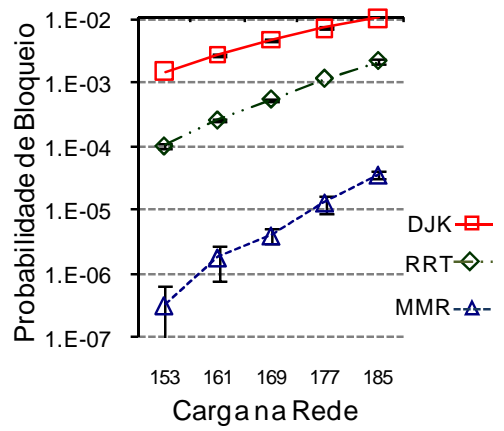

Fig. 3. Probabilidade de Bloqueio, EON - Tráfego Uniforme.

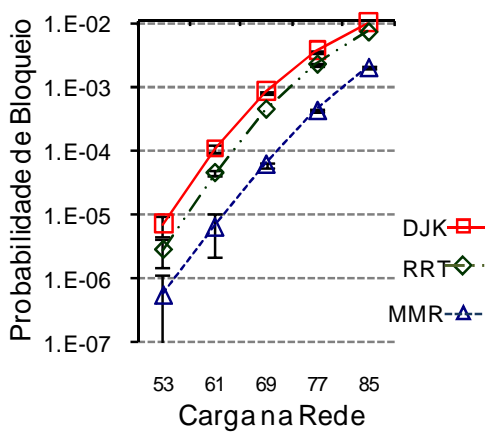

Fig. 4. Probabilidade de Bloqueio, Abilene - Tráfego Uniforme.

Observa-se (Figs. 2-4) que o desempenho relativo dos algoritmos é semelhante, independente da topologia utilizada. Nestes cenários, o algoritmo MMR apresenta um melhor desempenho, em termos de probabilidade de bloqueio, quando comparado com os outros algoritmos. Por outro lado, o algoritmo de menor caminho (Dijkstra) sempre 
apresenta um desempenho inferior em termos de probabilidade de bloqueio. Esses resultados são justificados pela capacidade do MMR em balancear a utilização dos enlaces da rede, utilizando apenas rotas de menor caminho [8].

A seguir são apresentados e analisados os resultados de avaliação de desempenho dos algoritmos sob tráfego não uniforme. As Figuras 5 a 7 exibem os gráficos de probabilidade de bloqueio considerando as topologias das redes RNP, EON e Abilene, respectivamente.

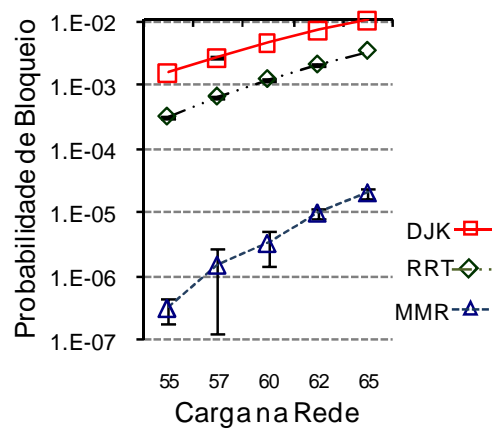

Fig. 5. Probabilidade de Bloqueio, RNP - Tráfego Não Uniforme.

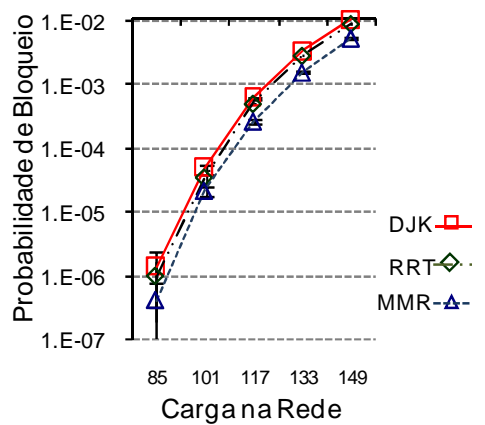

Fig. 6. Probabilidade de Bloqueio, EON - Tráfego Não Uniforme.

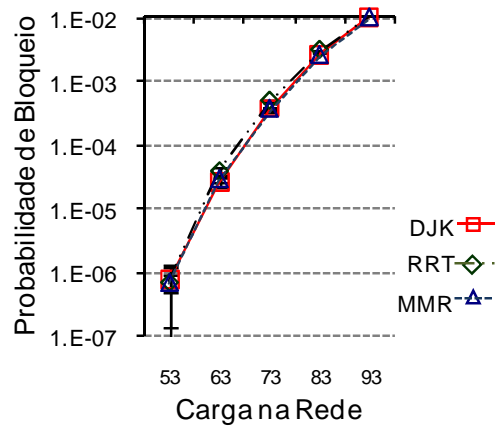

Fig. 7. Probabilidade de Bloqueio, Abilene - Tráfego Não Uniforme.

No cenário com a topologia da RNP (Fig. 5), observa-se que a probabilidade de bloqueio referente ao algoritmo de Dijkstra continuou sendo maior do que a probabilidade de bloqueio dos outros algoritmos. Nota-se também que o algoritmo RRT apresenta um melhor desempenho que o Dijkstra, em toda a faixa de carga de tráfego avaliada. Contudo, o MMR é o algoritmo que se destaca com menor probabilidade de bloqueio em relação aos outros algoritmos. Tal comportamento dos algoritmos com a topologia RNP sob tráfego não uniforme é semelhante ao observado no cenário sob tráfego uniforme. Isto mostra que o algoritmo MMR continuou eficaz em encontrar uma solução de rotas de menor caminho, além de balancear a utilização dos enlaces da rede.
Aplicando-se o algoritmo DJK no cenário RNP observouse pares de nós (origem, destino) com mais de $40 \%$ de bloqueio, enquanto que outros pares apresentaram zero de bloqueio refletindo um baixo nível de justiça (fairness). Por outro lado, aplicando-se o MMR, a máxima probabilidade de bloqueio é de 0,0007 . Isto mostra que o balanceamento proporcionado pelo MMR, não apenas diminuiu consideravelmente a probabilidade de bloqueio média geral, mas também atendeu as requisições de circuitos ópticos dos diversos pares de nós (origem, destino) com melhor justiça.

$\mathrm{O}$ gráfico de probabilidade de bloqueio referente à topologia EON (Fig. 6) mostra que, ao se utilizar o algoritmo MMR sob o tráfego não uniforme, não há um ganho tão significativo, como o ganho observado no mesmo cenário, sob tráfego uniforme (Fig. 3). A seguir é analisado como o tráfego não uniforme impacta no desempenho dos algoritmos de roteamento estudados. Esta análise é feita considerando o último ponto de carga aplicada na rede EON (Fig. 6), 149 Erlangs.

A Figura 8 mostra a probabilidade de bloqueio por nó de origem de requisições. Observa-se que as rotas de menor caminho encontradas pelo algoritmo MMR apresentam menor probabilidade de bloqueio para todos os nós de origem da rede. Contudo, aplicando o algoritmo MMR, o nó 19 apresenta mais de $2 \%$ de bloqueio, enquanto que os demais nós ficam com um valor abaixo de $0,2 \%$. Devido a este valor discrepante, a seguir o nó 19 é analisado de forma mais detalhada.

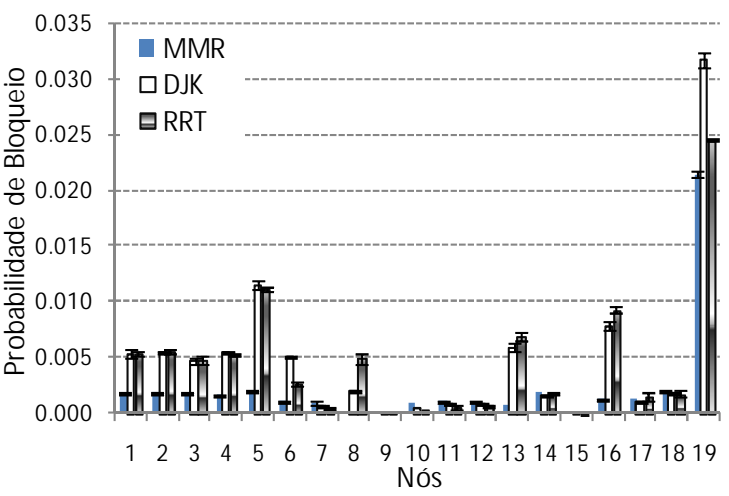

Fig. 8. Probabilidade de Bloqueio por Nó - EON.

A Figura 9 ilustra a probabilidade de bloqueio para cada par de nós (origem, destino), tendo como origem o nó 19. Neste gráfico nota-se que há um balanceamento da probabilidade de bloqueio quando se utiliza o MMR. Alguns pares que apresentam maior probabilidade de bloqueio com os algoritmos DJK e RRT, como os pares $(19,4),(19,1)$, $(19,3)$ e $(19,5)$, quando aplicado o MMR tiveram uma queda significativa na probabilidade de bloqueio. Para isto, alguns pares de nós que estavam mais "folgados" em termos de probabilidade de bloqueio, como por exemplo, os pares $(19,13),(19,7),(19,10)(19,12)$ e $(19,16)$, tiveram alguns enlaces de suas respectivas rotas compartilhados com um número maior de rotas, aumentando a probabilidade de bloqueio destes pares. Portanto, também neste cenário, pode-se dizer que o MMR é um algoritmo mais justo no que diz respeito ao atendimento das diversas requisições de circuitos ópticos.

Observa-se ainda na Figura 9 que não houve bloqueio 
para os pares $(19,15),(19,8)$ e $(19,9)$, independente do algoritmo de roteamento fixo aplicado. A seguir, é justificado o motivo pelo qual estes pares de nós não apresentam bloqueio.

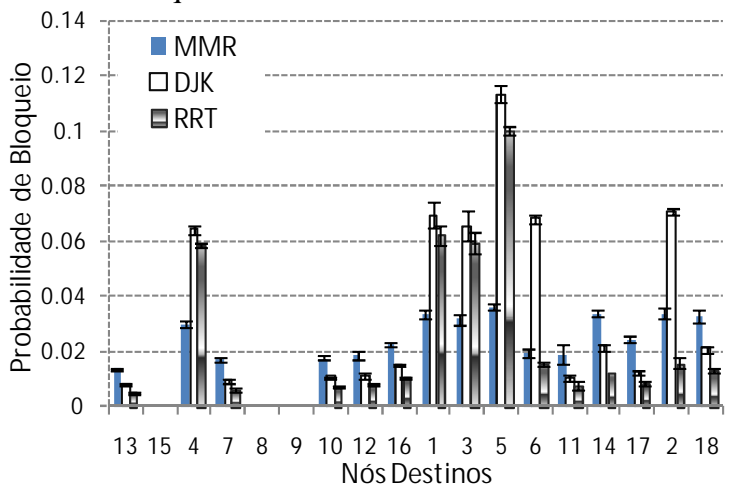

Fig. 9. Probabilidade de Bloqueio, Nó Origem 19 - topologia EON.

A Figura 10 ilustra a topologia da rede EON, destacando os nós de destino (do nó 19) que apresentam probabilidade de bloqueio igual a zero (nós 15, 8 e 9). Nota-se que o nó 19 possui apenas dois enlaces de saída. São eles: $\langle 19,15\rangle$ e $<19,13\rangle$. Aplicando o algoritmo MMR, apenas os nós de destino com zero de bloqueio utilizam o primeiro enlace, enquanto que o segundo enlace é compartilhado por todos os outros nós de destino da rede. Ele é o mais utilizado de toda a rede, alcançando uma taxa de $75 \%$ de utilização. Todas as rotas de menor caminho para cada um destes nós de destino utilizam o enlace $\langle 19,13\rangle$, totalizando uma carga de aproximadamente $11 \%$ de toda a rede. Com isso, uma vez que o algoritmo MMR sempre atribui rotas de menor caminho, ele não pode deixar de utilizar este enlace sobrecarregado, para utilizar o enlace $\langle 19,15\rangle$. Vale ressaltar que no modelo do tráfego não uniforme, os nós 19 , 13 e 4 são responsáveis por $42 \%$ de todo o tráfego gerado na rede. Contudo, diferentemente do nó 19, os nós 13 e 4 apresentam sete enlaces de saída, aumentando suas possibilidades de balanceamento de carga.

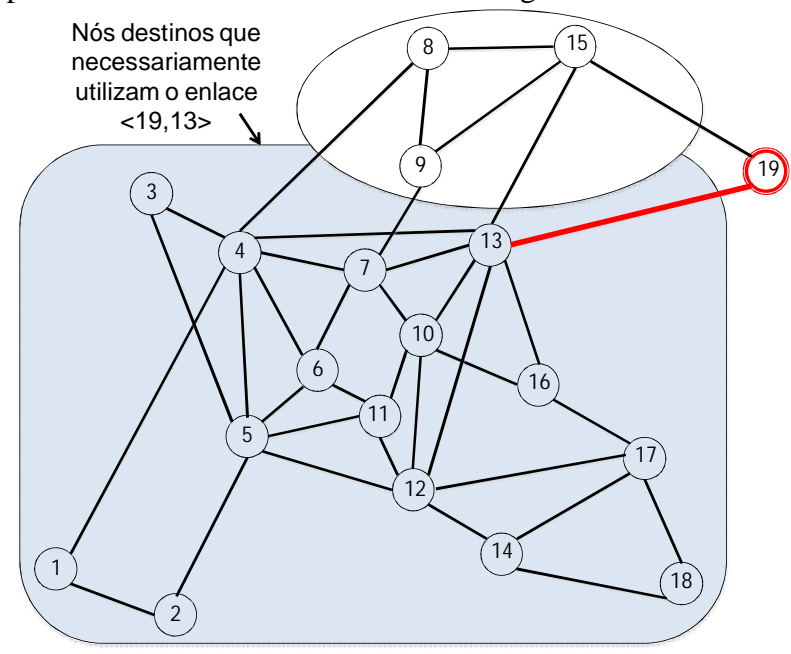

Fig. 10. Enlace sobrecarregado - EON.

Portanto, apesar do melhor desempenho do algoritmo MMR, justificado pela sua capacidade de balanceamento de carga, as condições de tráfego e topologia juntas, no cenário da rede EON, levaram a uma limitação deste desempenho.

No cenário da topologia Abilene (Fig. 7), o impacto do tráfego não uniforme no desempenho relativo dos algoritmos de roteamento é ainda maior.

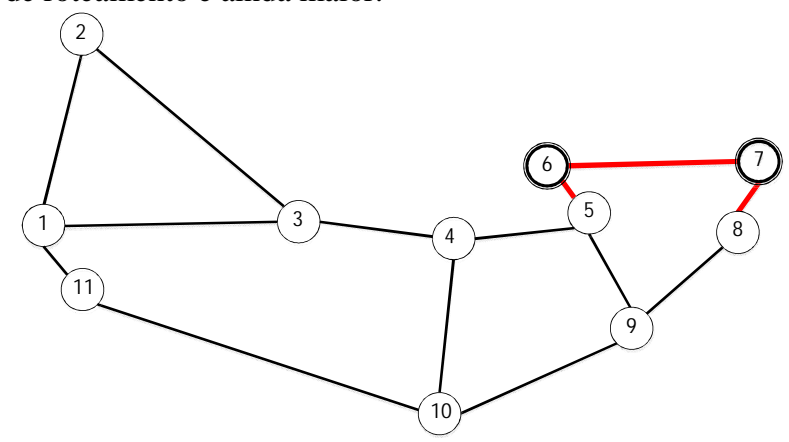

Fig. 11. Enlaces sobrecarregados - Abilene.

Metade do tráfego gerado nesta rede tem origem nos nós 6 e 7. Percebe-se que as rotas fixas definidas com origem nestes nós, necessariamente, devem utilizar os enlaces $\langle 5,6\rangle,\langle 6,7\rangle$ ou $\langle 7,8\rangle$, o que causa uma sobrecarga nestes enlaces (Fig. 11). Desta forma, as diferentes soluções de rotas obtidas com a execução dos algoritmos Dijkstra, RRT e MMR, apresentam desempenhos semelhantes em termos de probabilidade de bloqueio.

O gráfico da Fig. 12 representa a probabilidade de bloqueio de requisições de circuitos por nó de origem, considerando o ponto de carga de 93 Erlangs. Observa-se que o desempenho dos algoritmos continua semelhante em cada nó. Além disso, é notável que a maior parte do bloqueio ocorre em requisições com origem nos nós 6,7 e 8 . As Figuras 13, 14 e 15 mostram a probabilidade de bloqueio para cada destino, com origem nos nós 6,7 e 8 , respectivamente.

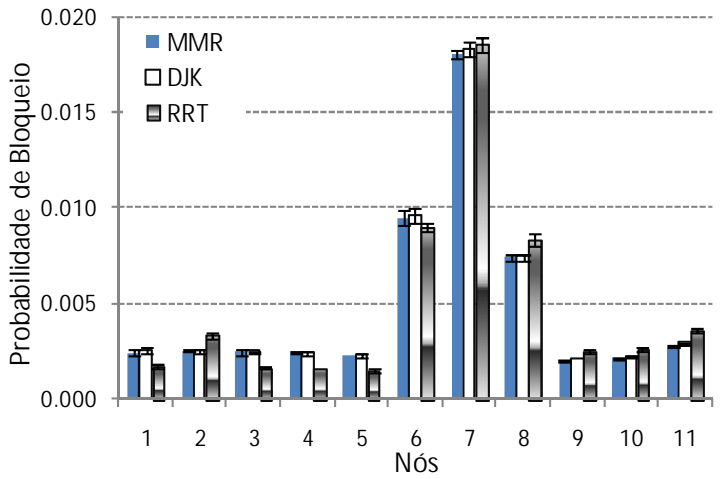

Fig. 12. Probabilidade de Bloqueio por Nó - Abilene.

A Figura 13 mostra que há certa uniformidade no atendimento das requisições de circuito com origem no nó 7 , à exceção das requisições com destino no nó 6 , as quais apresentam menor probabilidade de bloqueio. Esta menor probabilidade é justificada porque o nó 6 está diretamente conectado ao nó 7 e, além disso, as outras rotas que utilizam o enlace $<7,6>$ possuem em média um tamanho (número de saltos) igual a 3,5, favorecendo ao atendimento das requisições de circuito do par $(7,6)$, o qual possui apenas um salto. Apesar do nó 8 também estar diretamente conectado ao nó 7, o mesmo não ocorre com as requisições do par $(7,8)$. O tamanho médio das outras requisições de circuito que utilizam o enlace $\langle 7,8\rangle$ é de apenas 2,75. Estas requisições são mais atendidas (em comparação com aquelas de tamanho médio igual 3,5), dificultando o atendimento às requisições do par $(7,8)$. 


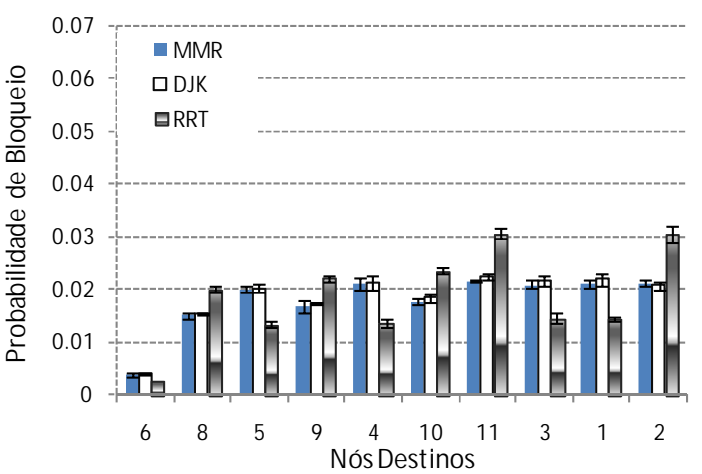

Fig. 13. Probabilidade de Bloqueio, Nó Origem 7 - topologia Abilene.

Para os demais pares com origem no nó 7,6 rotas definidas utilizam o enlace $\langle 7,6\rangle$ e 4 rotas utilizam o enlace $<7,8>$, considerando a configuração de rotas obtidas com o DJK ou MMR. A única rota diferente obtida com o RRT, é a rota 7-8-9-10-11-1-2 do par(7,2) que aumenta o número de saltos em uma unidade. Além disso, esta diferença diminui o número de rotas que utilizam o enlace $\langle 7,6\rangle$ e aumenta o número de rotas do enlace $\langle 7,8\rangle$, ficando ambos com 5 rotas cada. Com isto, no RRT, os 5 destinos que utilizam o enlace $<7,6>$ (nós 6, 5, 4, 3 e 1) apresentam um notável ganho na probabilidade de bloqueio. Em contrapartida, para os outros 5 destinos que utilizam o enlace $\langle 7,8\rangle$ (nós $8,9,10,11$ e 2) o desempenho em termos de probabilidade de bloqueio diminui.

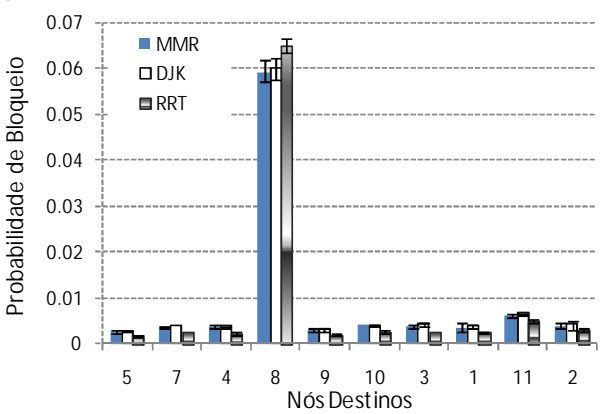

Fig. 14. Probabilidade de Bloqueio, Nó Origem 6 - topologia Abilene.

Tendo como origem o nó 6 , o nó 8 é o destino que se destaca com maior probabilidade de bloqueio de requisições, ficando em torno de 0,06 , enquanto que os demais pares de nós com origem no nó 6 estão abaixo de 0,01 (Fig. 14). Tanto o algoritmo MMR, como o DJK e o RRT, escolheram a rota 6-7-8 para o par $(6,8)$. Esta rota utiliza dois dos três enlaces sobrecarregados, os enlaces $\langle 6,7\rangle$ e $\langle 7,8\rangle$, enquanto que as outras rotas, com origem no nó 6 , utilizam apenas um enlace sobrecarregado, o enlace $<6,5\rangle$. Por isso, o nó 8 apresenta maior probabilidade de bloqueio.

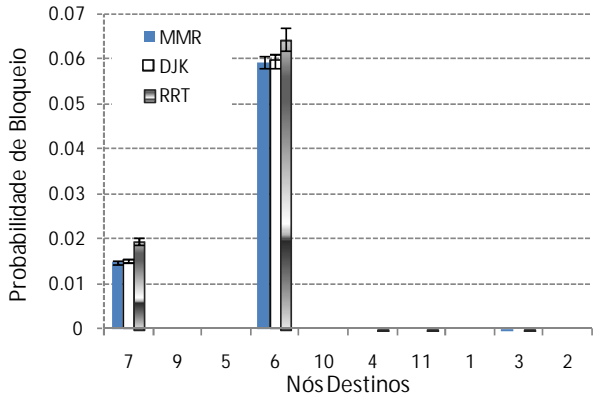

Fig. 15. Probabilidade de Bloqueio, Nó Origem 8 - topologia Abilene.
Por último, a Fig. 15 mostra o gráfico da probabilidade de bloqueio das requisições com origem no nó 8. É possível observar que praticamente só houve bloqueio para os nós destinos 7 e 6 . Isto ocorre porque apenas as rotas para estes destinos utilizam os enlaces sobrecarregados $\langle 8,7\rangle$ e $\langle 7,6\rangle$. Todas as rotas para os demais pares com origem no nó 8 não utilizam nenhum dos enlaces sobrecarregados, independente do algoritmo de roteamento aplicado.

\section{CONCLUSÕES}

Este trabalho apresentou um estudo do impacto do tráfego não uniforme no desempenho dos algoritmos de roteamento fixo em redes ópticas transparentes. Foram consideradas três topologias, nas quais foram observados diferentes comportamentos dos algoritmos em função das características de tráfego e da configuração topológica. Há cenários em que as características de tráfego ocasionam "gargalos" no desempenho dos algoritmos. Contudo, de maneira geral, o algoritmo MMR se destaca em termos de menor probabilidade de bloqueio em comparação com os outros algoritmos de roteamento fixo, nos cenários avaliados neste trabalho. Além disso, o MMR apresenta uma tendência em tratar com melhor justiça às diversas requisições de todos os pares de nós (origem, destino), mesmo sob o tráfego não uniforme.

\section{REFERÊNCIAS}

[1] E. Palkopolou; D.A. Schupke; C.G. Gruber; A. Kirstädter e C. Merkle, "Service Oriented Traffic Models for Future Backbone Networks", ITG-Fachtagung Photonische Netze, Leipzig, Alemanha, Maio 2007.

[2] C. S. R. Murthy e M. Gurusamy, WDM Optical Networks - Concepts, Design and Algorithms. Prentice Hall PTR, 2002.

[3] H. Zang, J. P. Jue e B. Mukherjee, "A Review of Routing and Wavelength Assignment Approaches for Wavelength-Routed Optical WDM Network". Optical Network Magazine, 2000.

[4] E. W. Dijkstra. "A Note on Two Problems in Connection with Graphs". Numerical Mathematics, 1: 269-271, 1959.

[5] H. Harai, M. Murata, e H. Miyahara, "Performance of Alternate Routing Methods in All-optical Switching Networks," in Proceeding of IEEE INFOCOM 1997, vol. 2, pp. 516 - 524, Abril 1997.

[6] H. C. Lin, S. W. Wang e C. Tsai, "Traffic Intensity Based FixedAlternate Routing in All-Optical WDM Networks", in Proceedings of the IEEE ICC'2006, Istanbul, Turkey, June 11 - 15, 2006.

[7] P. Rajalakshmi e A.Jhunjhunwala, "Load Balanced Routing to Enhance the Performance of Optical Backbone Networks", in 5th IFIP International Conference on Wireless and Optical Communications Networks (WOCN 2008), Surabaya, Indonésia, 2008.

[8] G. M. Durães, A. C. B. Soares e W. F. Giozza, "A Escolha da Melhor entre as Menores Rotas em Redes Ópticas Transparentes" in Anais do 27 Simpósio Brasileiro de Redes de Computadores e Sistemas Distribuídos - SBRC 2009, Recife, Maio 2009.

[9] A. C. B. Soares, G. M. Durães, W. F. Giozza e P. Cunha, "TONetS: Ferramenta para Avaliação de Desempenho de Redes Ópticas Transparentes" in VII Salão de Ferramentas do Simpósio Brasileiro de Redes de Computadores - SBRC, Maio 2008.

[10] M. J. O'Mahony, M. C. Sinclair e B. Mikac, "Ultra-High Capacity Optical Transmission Network: European Research Projetct COST 239" in Information, Telecommunications, Automata Journal, v.12 n.1-3, pp.33-45, Croácia, 1993.

[11] "Rede Nacional de Ensino e Pesquisa - RNP". Disponível em: http://www.rnp.br/backbone, acessado em 07/04/2009.

[12] M. J. Mahony, "A European optical network: design considerations" in IEEE Colloquium on Transparent Optical Networks, p. 1-16, Abril 1994

[13] "Abilene Network". Disponível em: http://ieeexplore.ieee.org/ search/wrapper.jsp?arnumber $=4448651$, acessado em 07/04/2009. 